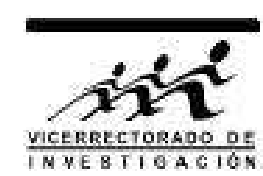

\title{
Estimación del espesor óptico de los aerosoles a partir de las imágenes del sensor MODIS sobre el Perú (2004-2005)
}

\author{
E. Cholan, J. Rojas, B. L. Willems y L. C. Ocola \\ Facultad de Ciencias Físicas, Universidad Nacional Mayor de San Marcos, Lima, Perú
}

Recibido 20 noviembre 2016 - Aceptado 15 diciembre 2016

\begin{abstract}
En el presente trabajo estimamos el espesor óptico de los aerosoles, Aerosols Optical Thickness-AOT-, en la longitud de onda de $0.55 \mu \mathrm{m}$ usando las imágenes mod021 km - level $1 \mathrm{~b}$ calibrated radiances $-1 \mathrm{~km}$ como entrada del modelo de transferencia radiativa SBDART [1]. El área de estudio es el Perú en las latitudes geográficas 0 a $20^{\circ} \mathrm{S}$ y las longitudes geográficas de 82 a $68^{\circ} \mathrm{O}$. El modelo SBDART simula la transferencia radiativa en la atmósfera (scattering, absorción, emisión, etc.), estimando la irradiancia en el tope de la atmósfera y en la superficie terrestre. La aproximación de dos flujos y la aproximación de Eddington son usados para resolver la ecuación de transferencia radiativa considerando una atmósfera plano paralela. Mediante este modelo se generan las ecuaciones definidas para el AOT de $0.55 \mu \mathrm{m}$ en función de la reflectancia en el tope de la atmósfera para la banda 1 , de $0.66 \mu \mathrm{m}$ del sensor MODIS.

EI AOT de $0.55 \mu \mathrm{m}$ estimado por el modelo SBDART se verifica con los datos del AOT de $0.55 \mu \mathrm{m}$ medidas in-situ en la estación de la red AERONET ubicada en Rio Branco, Brasil, tanto para el año 2004 y 2005. La estación se encuentra en la latitud $9.954^{\circ} \mathrm{S}$ y longitud $67.869^{\circ} \mathrm{O}$. La gráfica de la dispersión de las series de tiempo del AOT para ambas observaciones muestran una correlación (no) significativa de 0.99 (0.06) para los año 2004 y 2005.

También se verifica el AOT obtenido de las imágenes MODIS (MOD04_L2) con el AOT estimado por el modelo SBDART, la gráfica de dispersión de las series de tiempo de ambos presentan una correlación significativa de 0.99 para el 2004 y 2005, mientras que la verificación del AOT de las imágenes MODIS (MOD04 L2) con los datos del AOT de $0.55 \mu \mathrm{m}$ in-situ de Rio Branco, muestra una gráfica de dispersión de las series de tiempo con una correlación (no) significativa de 0.98 (0.02) para los años 2004 y 2005.

El modelo SBDART utilizado para la estimación del AOT se limita para la parte central y sur del Perú, debido a su ubicación geográfica, las condiciones meteorológicas, la circulación atmosférica y a las propiedades ópticas de los aerosoles.

Palabras claves: SBDART, MODIS, aerosoles, espesor óptico, AERONET.
\end{abstract}

\section{Aerosols optical thickness estimation from MODIS sensor images over Perú (2004-2005)}

In the present work we estimate the aerosols optical thickness (AOT) in the wavelength of $0.55 \mu \mathrm{m}$ using the mod021 km - level 1B Calibrated Radiances - $1 \mathrm{~km}$ images as input of the SBDART radiative transfer model [1. The study area is Peru in the geographic latitudes 0 to $20^{\circ} \mathrm{S}$ and the geographic longitudes of 82 to $68^{\circ} \mathrm{O}$. The SBDART model simulates the radiative transfer in the atmosphere (scattering, absorption, emission, etc.), estimating the irradiance in the top of the atmosphere and on the Earth surface. The two-stream and Eddington approximations are used to solve the radiative transfer equation, considering a parallel plane atmosphere. This model generate equations defining the AOT of $0.55 \mu \mathrm{m}$ as a function of the reflectance at the top of the atmosphere (TOA) for the band 1 at $0.66 \mu \mathrm{m}$ of the MODIS sensor.

The $0.55 \mu \mathrm{m}$ AOT estimated by the SBDART model was verify with the $0.55 \mu \mathrm{m}$ AOT data measured in-situ from an AERONET network station located in Rio Branco, Brazil, for the both year 2004 and 2005. The station is located at latitude $9.954^{\circ} \mathrm{S}$ and longitude $67.869^{\circ} \mathrm{O}$. The dispersion dataplot of the time series of both AOT showed a (not) significant correlation of 0.99 (0.06) for the year 2004 and 2005.

Also, was verify the AOT obtained from the MODIS images (MOD04_L2) with the AOT estimated by the SBDART model, the scatter plot of the time series of both AOT showed a significant correlation of 0.99 for both year 2004 and 2005, while the verification of the AOT from the MODIS images (MOD04_L2) with the 0.55 $\mu \mathrm{m}$ AOT data in-situ from Rio Branco, show in the dispersion dataplot of the time series an (not) significant correlation of 0.98 (0.02) for the year 2004 and 2005.

The SBDART model used for estimating the AOT is limited to the Central and Southern part of Peru, due to its geographic location, meteorological conditions, atmospheric circulation and optical properties of aerosols.

Keywords: SBDART, MODIS, aerosols, optical thickness, AERONET.

${ }^{*}$ echolanr@unmsm.edu.pe 
Los aerosoles atmosféricos se definen como partículas en estado sólido o líquido que se encuentran suspendidas en el aire que se mueve y son arrastradas por éste. Su tamaño es muy variable, con radios situados entre $0.001 \mu \mathrm{m}$ y $100 \mu \mathrm{m}$, es decir, desde agregados moleculares hasta partículas masivas que por su tamaño sólo pueden permanecer suspendidas en la atmósfera durante unas pocas horas [2].

Los aerosoles atmosféricos pueden originarse de manera natural, aerosoles biogénicos, o pueden ser producidos por la actividad humana, aerosoles antropogénicos. Las principales fuentes biogénicas son el suelo y los restos de roca, polvo natural, erupciones volcánicas, brisas marinas atomizadas, quema de biomasa, incendios forestales y reacciones entre las emisiones naturales. Los aerosoles antropogénicos provienen de cuatro fuentes principales: combustión, procesos industriales, fuentes fugitivas no industriales como el polvo de carreteras pavimentadas y destapadas, erosión de las tierras de cultivo, construcciones y fuentes relacionadas con el transporte aéreo y terrestre [3]. Los aerosoles son un constituyente importante de la atmósfera, que interaccionan de forma directa e indirecta con la radiación solar y terrestre, afectando al balance radiativo Tierra-Atmósfera. De forma directa, los aerosoles absorben y dispersan la radiación proveniente del Sol en su paso a través de la atmósfera. Como el tamaño de estas partículas es similar al de la longitud de onda de la radiación solar, los aerosoles pueden interactuar eficazmente con ésta, reduciendo la irradiancia solar total que alcanza la superficie de la Tierra. De forma indirecta, los aerosoles actúan como núcleos de condensación (CCN) en la formación de nubes, lo que produce un aumento de la reflectancia de la nube para la radiación de onda corta, pero muy poca o ninguna variación para la radiación de onda larga. Esta influencia de los aerosoles sobre el balance radiativo Tierra-Atmósfera se conoce como el forzamiento radiativo de los aerosoles, y su efecto puede ser de la misma magnitud pero de sentido opuesto que el forzamiento radiativo debido a los gases de efecto invernadero [4].

Ante la evidencia de estos efectos sobre el clima, en los últimos años el estudio de los aerosoles atmosféricos ha cobrado gran interés, desarrollándose nuevos instrumentos y metodologías para su estudio [5]. Por ejemplo, las masas de aire contaminado que provienen de las quemas de biomasa en Mato Grosso, Brasil, del Norte Amazónico de Bolivia y Paraguay y que se suman a las quemas locales, contaminan la atmósfera peruana debido a las corrientes de viento que se dirigen de este a oeste, hacia el Ecuador [6], influyendo en la variabilidad del AOT en la longitud de onda de $0.55 \mu \mathrm{m}$ en la atmósfera. Con el objetivo de obtener una mejor visualización de la distribución espacial de los AOTs en la longitud de onda de $0.55 \mu \mathrm{m}$ para el Perú, estimamos, en este trabajo, con una resolución espacial de $1 \mathrm{~km} \times 1 \mathrm{~km}$ empleando el modelo de transferencia radiativa SBDART.

\section{Teoría}

El SBDART, en primer lugar, es el acrónimo en inglés de Santa Barbara Disort Atmospheric Radiative Transfer, puesto que miembros de la Universidad de California en Santa Barbara desarrollaron un software que calcula la transferencia radiativa en planos paralelos en condiciones de nubosidad y de claridad dentro de la atmósfera y sobre la superficie terrestre. Todos los procesos importantes que afectan los campos de radiación en el ultrvioleta, visible e infrarrojo son incluídos. El código está enlazado a un módulo de transferencia radiativa con variables discretizadas, a modelos de transmisión atmosféricos de baja resolución y a los resultados del scattering de Mie para el scattering de luz producidos por las gotas de agua y cristales de hielo. El código está bien estructurado para una amplia variedad de balance de energía radiativa atmosférica y estudios de sensoramiento remoto [1].

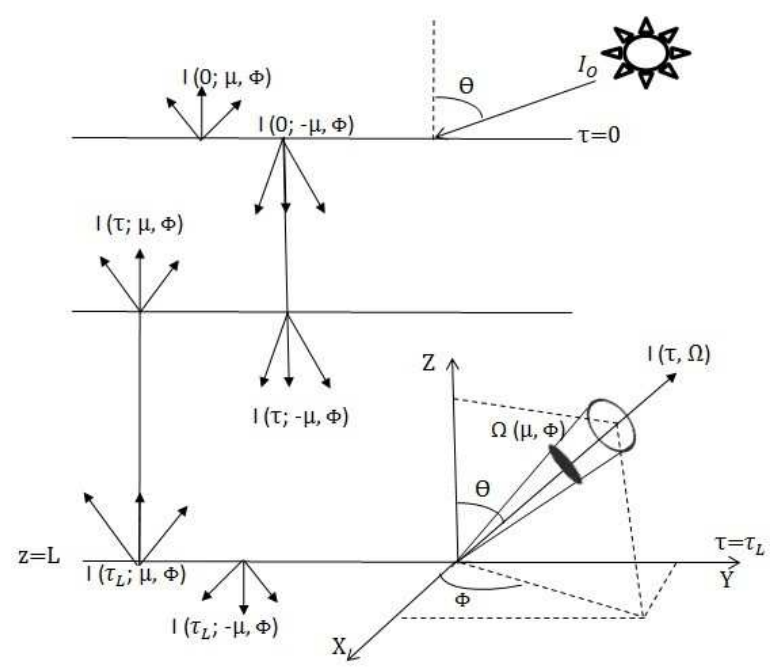

Figura 1: Geometría de una atmósfera plana paralela donde $\theta$ y $\Phi$ son los ángulos cenit y acimut, respectivamente [7].

Algunas de sus características resaltantes son: a) Está desarrollado íntegramente en lenguaje Fortran 77. b) Es un modelo de planos paralelos y permite un máximo de $65 \mathrm{ca}-$ pas. c) Tiene un archivo principal de entrada (atms.dat), el cual contiene información de la distribución de la temperatura, presión, densidad de vapor de agua y la densidad del ozono en toda la atmósfera. d) Puede introducirse datos asociados con nubes, por ejemplo: espesor óptico, cantidad de agua líquida, tamaño de las gotas, etc.

El modelo de transferencia radiativa SBDART, resuelve la ecuación de transferencia radiativa, considerando una atmósfera plano paralela con o sin presencia de nubes. Para la resolución de la Ecuacion de Transferencia Radiativa, ETR, el programa está basado en el código DISORT, acrónimo en inglés de Discret Ordinate Radiative Transfer. Este código presenta las ecuaciones exactas de la ETR utilizando 
las aproximaciones de Eddington [1].

Las ecuaciones inversas están definidas por el espesor óptico en función de la reflectancia en el tope de la atmósfera, el modelo de transferencia radiativa SBDART genera un archivo de texto de salida en ASCII, que contiene la irradiancia en el tope de la atmósfera obtenido mediante la aproximación de dos flujos y la aproximación de Eddington para resolver la ecuación de transferencia radiativa, para una atmósfera plano paralela.

La ecuación escalar básica para la transferencia de radiación en una atmósfera plano paralela, denotando la radiancia por $I(z ; \theta, \Phi)$, ver Figura 1 , la función fuente por $J(z ; \theta, \Phi)$ y el espesor óptico de los aerosoles [7] por

$$
\tau=\int_{z}^{\infty} k \rho d z
$$

se puede escribir como

$$
\mu \frac{I(\tau ; \mu, \Phi)}{d \tau}=I(\tau ; \mu, \Phi)-J(\tau ; \mu, \Phi)
$$

donde $\mu=\cos (\theta)$.

$$
\begin{gathered}
J(\tau ; \mu, \Phi)=\frac{\widetilde{\omega}}{4 \pi} \int_{0}^{2 \pi} \int_{-1}^{1} I\left(\tau^{\prime} ; \mu^{\prime}, \Phi^{\prime}\right) \times \\
P\left(\mu, \Phi ; \mu^{\prime}, \Phi^{\prime}\right) d \mu^{\prime} d \Phi^{\prime}+\frac{\widetilde{\omega}}{4 \pi} F_{\odot} P\left(\mu, \Phi ;-\mu_{0},-\Phi_{0}\right) \times \\
e^{-\tau / \mu_{0}}+(1-\widetilde{\omega}) B[T(\tau)]
\end{gathered}
$$

La función fase puede ser numéricamente expandido en polinomios de Legendre con un número finito de términos, $N$. Los polinomios de Legendre pueden expandirse por el teorema de los armónicos esféricos como

$$
\begin{aligned}
& P\left(\mu, \phi ; \mu^{\prime}, \phi^{\prime}\right)=\sum_{m=0}^{N} \sum_{l=0}^{N} \widetilde{\omega}_{l}^{m} P_{l}^{m}(\mu) P_{l}^{m}\left(\mu^{\prime}\right) \cos m\left(\phi^{\prime}-\phi\right) \\
& \widetilde{\omega}_{l}^{m}=\left(2-\delta_{0, m}\right) \widetilde{\omega}_{l} \frac{(l-m) !}{(l+m) !}(l=m, \ldots, N \quad 0 \leq m \leq N) \\
& \delta_{0, m}= \begin{cases}1 & \text { si } m=0 \\
0 & \text { otros casos }\end{cases}
\end{aligned}
$$

donde $P_{l}^{m}$ denota los polinomios de Legendre. Expandiendo la intensidad en la forma

$$
I(\tau ; \mu, \phi)=\sum_{m=0}^{N} I^{m}(\tau, \mu) \cos m\left(\phi_{0}-\phi\right)
$$

para el caso independiente acimutal, la función fase será definida como

$$
\begin{aligned}
P\left(\mu, \mu^{\prime}\right) & =\frac{1}{2 \pi} \int_{0}^{2 \pi} P\left(\mu, \phi ; \mu^{\prime}, \phi^{\prime}\right) d \phi^{\prime} \\
& =\sum_{l=0}^{N} \widetilde{\omega}_{l} P_{l}(\mu) P_{l}\left(\mu^{\prime}\right)
\end{aligned}
$$

teniendo en cuenta la ecuación 6 , la ecuación 2 , quedara definida para el caso independiente acimutal $m=0$ como [7]

$$
\begin{gathered}
\mu \frac{d I(\tau, \mu)}{d \tau}=I(\tau, \mu)-\frac{\widetilde{\omega}}{2} \int_{-1}^{1} I\left(\tau ; \mu^{\prime}\right) P\left(\mu, \mu^{\prime}\right) d \mu^{\prime} \\
\left.+\frac{\widetilde{\omega}}{4 \pi} F_{\odot} P\left(\mu,-\mu_{0}\right) e^{-\tau / \mu_{0}}+(1-\widetilde{\omega}) B[T(\tau))\right] .
\end{gathered}
$$

Las densidades de flujo solar difuso hacia arriba y hacia abajo están dados respectivamente por

$$
\begin{aligned}
& F_{d i f}^{\uparrow}(\tau)=\int_{0}^{2 \pi} \int_{0}^{1} I(\tau ; \mu, \phi) \mu d \mu d \phi, \quad \mu \geq 0 \\
& F_{d i f}^{\downarrow}(\tau)=\int_{0}^{2 \pi} \int_{0}^{-1} I(\tau ; \mu, \phi) \mu d \mu d \phi, \quad \mu \leq 0
\end{aligned}
$$

para resolver analíticamente la ecuación 2 , reemplazaremos la integral por una sumatoria sobre un numero finito de puntos de cuadratura de Gauss. La integración gaussiana para cualquier función $f(\mu)$ está dado por

$$
\int_{-1}^{1} f(\mu) d \mu \approx \sum_{j=-n}^{n} a_{j} f\left(\mu_{j}\right)
$$

donde los pesos son

$$
a_{j}=\frac{1}{P_{2 n}^{\prime}\left(\mu_{j}\right)} \int_{-1}^{1} \frac{P_{2 n(\mu)}}{\mu-\mu_{j}} d \mu
$$

donde $\mu_{j}$ son los ceros de los polinomios de Legendre de orden par $P_{2 n}(\mu)$, y los primados denotan la diferenciación con respecto a $\mu_{j}$. Empleando la cuadratura de Gauss, la ecuación 7 , puede ser escrita como

$$
\begin{gathered}
\mu_{i} \frac{d I\left(\tau, \mu_{i}\right)}{d \tau}=I\left(\tau, \mu_{i}\right)-\frac{\widetilde{\omega}}{2} \sum_{l=0}^{N} \widetilde{\omega}_{l} P_{l}\left(\mu_{i}\right) \sum_{j=-n}^{n} a_{j} P_{l}\left(\mu_{j}\right) \times \\
I\left(\tau, \mu_{j}\right)-\frac{\widetilde{\omega}}{4 \pi} F_{\odot}\left[\sum_{l=0}^{N}(-1)^{l} \widetilde{\omega}_{l} P_{l}\left(\mu_{i}\right) P_{l}\left(\mu_{0}\right] e^{-\tau / \mu_{0}}\right.
\end{gathered}
$$

donde $\mu_{i}(-n, n)$ representa las direcciones de los flujos de radiación. Reordenando términos y denotando $I^{\uparrow}=$ $I\left(\tau, \mu_{1}\right)$ y $I^{\downarrow}=I\left(\tau,-\mu_{1}\right)$. Mediante un sencillo análisis se obtiene

$$
\begin{aligned}
& I^{\downarrow}=I\left(\tau,-\mu_{1}\right)=K u e^{k \tau}+H v e^{-k \tau}+\gamma e^{-\tau / \mu_{0}} \\
& I^{\uparrow}=I\left(\tau, \mu_{1}\right)=K v e^{k \tau}+H u e^{-k \tau}+\varepsilon e^{-\tau / \mu_{0}}
\end{aligned}
$$

las constantes $K$ y $H$ son determinados de las condiciones de frontera de la radiación difusa en la parte superior e inferior de la capa de dispersión. Para introducir las aproximaciones de Eddington La intensidad dispersada puede 
expandirse en términos de los polinomios de Legendre como [7],

$$
I(\tau, \mu)=\sum_{l=0}^{N} I_{l}(\tau) P_{l}(\mu) .
$$

Para $N=1$ la función fase y intensidad pueden escribirse como

$$
\begin{aligned}
P\left(\mu, \mu^{\prime}\right) & =1+3 g \mu \mu^{\prime} \\
I(\tau, \mu) & =I_{0}(\tau)+I_{1}(\tau) \mu, \quad-1 \leq \mu \leq 1 .
\end{aligned}
$$

Los flujos difusos hacia arriba y hacia abajo son dados por

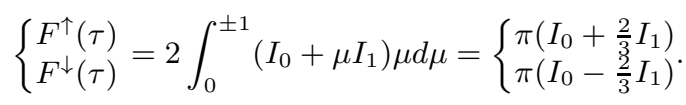

Utilizando la ecuación de transferencia radiativa denotada en la ecuación 10 y las densidades de flujo hacia arriba y hacia abajo definido en las ecuaciones 11,12 y omitiendo las contribuciones de la emisión, nosotros podemos escribir las siguientes ecuaciones generalizando la aproximación de dos flujos podemos expresar

$$
\begin{aligned}
& \frac{d F^{\uparrow}(\tau)}{d \tau}=\gamma_{1} F^{\uparrow}(\tau)-\gamma_{2} F^{\downarrow}(\tau)-\gamma_{3} \widetilde{\omega} F_{\odot} e^{-\tau / \mu_{0}} \\
& \frac{d F^{\downarrow}(\tau)}{d \tau}=\gamma_{2} F^{\uparrow}(\tau)-\gamma_{1} F^{\downarrow}(\tau)+\left(1-\gamma_{3}\right) \widetilde{\omega} F_{\odot} e^{-\tau / \mu_{0}}
\end{aligned}
$$

donde los coeficientes están dados en la siguiente tabla

\begin{tabular}{cccc}
\hline Método & $\gamma_{1}$ & $\gamma_{2}$ & $\gamma_{3}$ \\
\hline 2-stream & {$[1-\tilde{\omega}(1+g) / 2] / \mu_{1}$} & $\tilde{\omega}(1-g) / 2 \mu_{1}$ & $\left(1-3 g \mu_{1} \mu_{0}\right) / 2$ \\
\hline Eddington & {$[7-(4+3 g) \tilde{\omega}] / 4$} & $-[1-(4-3 g) \tilde{\omega}] / 4$ & $\left(2-3 g \mu_{0}\right) / 4$ \\
\hline
\end{tabular}

Sus respectivas soluciones están dadas por

$$
\begin{aligned}
& F^{\uparrow}=v K e^{k \tau}+u H e^{-k \tau}+\varepsilon e^{-\tau / \mu_{0}} \\
& F^{\downarrow}=u K e^{k \tau}+v H e^{-k \tau}+\gamma e^{-\tau / \mu_{0}} .
\end{aligned}
$$

Las condiciones de frontera estándar para determinar $K$ y $H$ son [8]

$$
\begin{aligned}
& F^{\uparrow}(\tau)=0 \\
& F^{\downarrow}(0)=0
\end{aligned}
$$

de donde obtenemos la reflectancia en el tope de la atmósfera

$$
R=\frac{F^{\uparrow}(0)}{F^{\downarrow}(0)}=\frac{\gamma_{2}[1-\exp (-2 k \tau)]}{k+\gamma_{1}+\left(k-\gamma_{1}\right) \exp (-k \tau)}
$$

donde $k^{2}=\gamma_{1}^{2}-\gamma_{2}^{2}$.

\section{Metodología}

El área de estudio corresponde a todo el territorio del Perú. Esta se encuentra ubicada en la parte central y occidental de América del Sur. El extremo septentrional del territorio peruano se encuentra en la parte superior de la primera curva que describe el rio Putumayo, al nororiente del poblado peruano de Guepi a $0^{\circ} 02^{\prime} 00^{\prime \prime} \mathrm{S}$, el extremo meridional se encuentra a orillas del mar en Tacna a $18^{\circ} 21^{\prime} 03^{\prime \prime}$ $\mathrm{S}$; el extremo oriental está en el rio Heath en Madre de Dios $68^{\circ} 39^{\prime} 00^{\prime \prime} \mathrm{O}$ y el extremo occidental se encuentra en Punta Balcones en Piura a $81^{\circ} 19^{\prime} 35^{\prime \prime} \mathrm{O}$.

Se descarga el producto MODIS L1B (MOD021KM) a partir de la ventana principal del sistema de distribución y el archivo de atmósfera y nivel 1 (LAADS). Los productos MODIS L1B (MOD021KM) se encuentran en el formato de datos HDF, Hierarchical Data Format. El prefijo MOD es reservado para archivos que contienen datos recogidos por la plataforma TERRA [9].

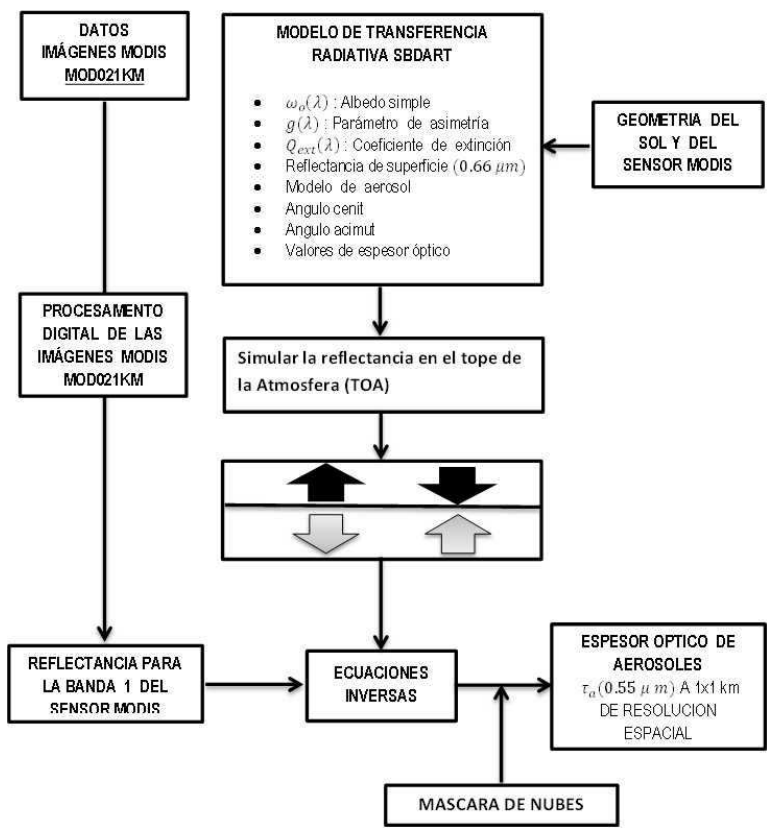

Figura 2: Diagrama del proceso de cálculo del AOT usando el código SBDART [1], en la longitud de onda de $0.55 \mu \mathrm{m}$ a 1 $\mathrm{km} \times 1 \mathrm{~km}$ de resolución espacial.

Se estima la radiancia en unidades de $\mathrm{Wm}^{-2} \mathrm{sr}^{-1} \mu \mathrm{m}^{-1}$ y la reflectancia en el tope de la atmósfera a partir de una imagen MODIS Level 1B. Además se emplea el modelo de transferencia radiativa SBDART [1, el cual genera un archivo de salida ASCII, que contiene la irradiancia en el 
tope de la atmósfera y en la superficie terrestre, obtenido mediante la aproximación de dos flujos (2-streams) y la aproximación de Eddington para resolver la Ecuación de Transferencia Radiativa, considerando una atmósfera plano paralela. Para luego generar las ecuaciones inversas definidas por el AOT en la longitud de onda de $0.55 \mu \mathrm{m}$ en función de la reflectancia en el tope de la atmósfera, mediante una regresión polinomial de grado 3 , en la longitud de onda de $0.66 \mu \mathrm{m}$ correspondiendo a la banda 1 de la imagen LEVEL 1B (MOD021KM) del sensor MODIS, con diferentes valores de AOT en la longitud de onda de $0.55 \mu \mathrm{m}$ y otras condiciones tales como la geometría del Sol y del sensor, un modelo de aerosoles y la reflectancia de la superficie terrestre en la longitud de onda de $0.66 \mu \mathrm{m}$ correspondiendo a la banda 1 de la imagen LEVEL 1B (MOD021KM) del sensor MODIS.

Finalmente luego de haber generado las ecuaciones inversas definidas por el AOT en función de la reflectancia en el tope de la atmósfera en la longitud de onda de 0.66 $\mu \mathrm{m}$ correspondiendo a la banda 1 de la imagen LEVEL $1 B$ (MOD021KM) del sensor MODIS, se aplica una máscara de nubes obtenido a partir del producto MOD035 con el objetivo de que la imagen quede libre de nubes, ver Figura 3.

Las informaciones mantenidas constantes en todos los cálculos son Flujo Solar Lowtran 7 con alta resolución, el factor de distancia solar $0.9688 \mathrm{UA}^{-2}$, no se utilizaron filtros espectrales correspondientes al sensor, pero las longitudes de onda fijas en $\lambda_{c}=66 \mu \mathrm{m}$ y $\lambda_{c}=2.1 \mu \mathrm{m}$, las condiciones atmosféricas son definidas por el modelo tropical, sin embargo la presión se fija en la superficie a $935 \mathrm{mb}$ y el contenido de vapor de agua en la columna $1.5 \mathrm{~cm}$ [10]. Los parámetros de entrada que se utilizaron como las geometrías del Satélite y del Sol, esto es, los ángulos cenital $\left(\theta_{0}, \theta\right)$ y acimutal ( $\phi$ SBDART), fueron para cada uno de los días considerados. Se define un modelo de aerosoles a partir de las propiedades ópticas $\left(\omega_{0}, g, Q_{\text {ext }}\right)$ obtenidos a partir de una estación de la red AERONET ubicada en São Paulo [10], ver Figura 4.

\section{Resultados y discusión}

La variabilidad espacial y temporal de las AOTs en la longitud de onda de $0.55 \mu \mathrm{m}$, se estiman para una resolución espacial de $1 \mathrm{~km} \times 1 \mathrm{~km}$, mediante el código de SBDART [1], para todos los casos estudiados. Por cuestiones de espacio y síntesis denominaremos a estos resultados como AOTs SBDART. A los resultados de AOTs obtenidos de los datos recolectados in situ por la red AERONET, los denominaremos de AOTs AERONET y para los AOTs obtenidos por los datos proporcionados por la NASA para los AOTs en la longitud de onda de $0.55 \mu \mathrm{m}$ y con una resolución espacial de $10 \mathrm{~km} \times 10 \mathrm{~km}$ los denominaremos AOTs NASA.

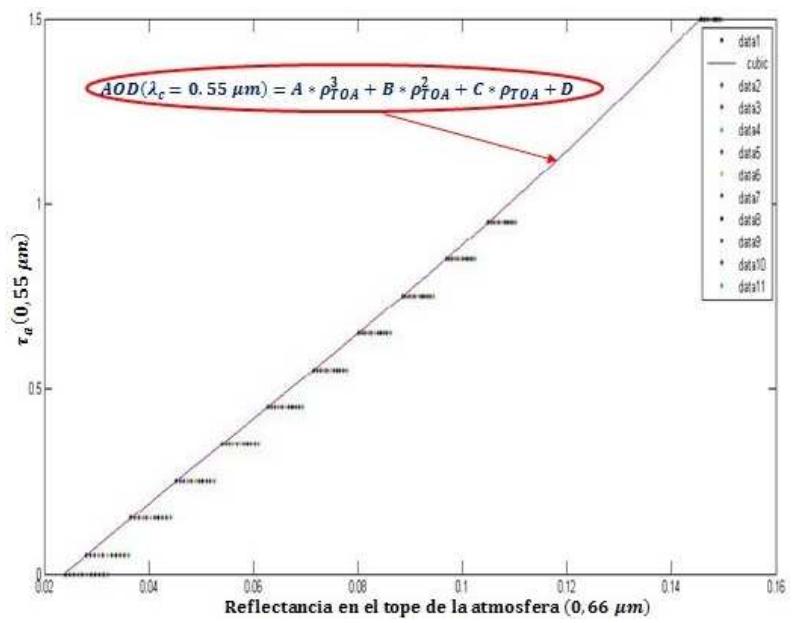

(a)

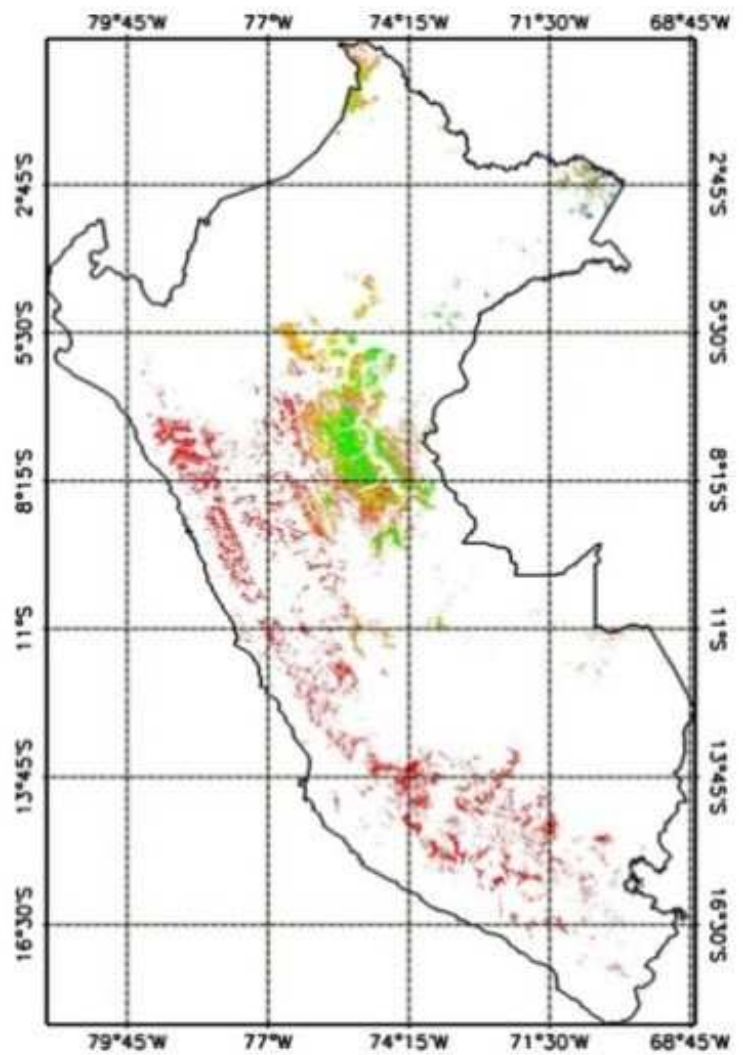

(b)

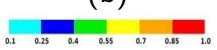

Figura 3: En (a) se muestran el AOT a $0.55 \mu \mathrm{m}$ en función de la reflectancia en el tope de la atmósfera, obtenidas por las imágenes del sensor MODIS en la longitud de onda de $0.66 \mu \mathrm{m}$ y con el detalle mencionado en el texto. En (b), se muestra el AOT a $0.55 \mu \mathrm{m}$ con una resolución espacial de $1 \mathrm{~km} \times 1 \mathrm{~km}$ Para todo el territorio del Perú, 02/01/2005. 


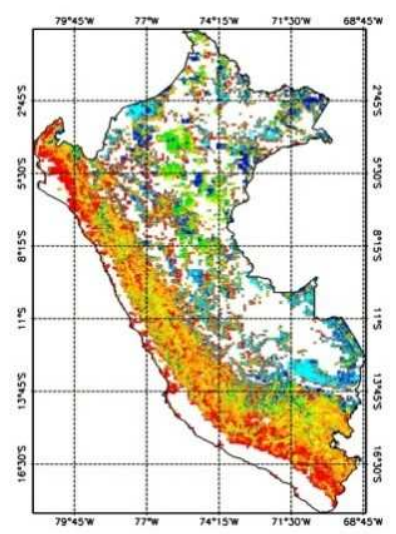

(a)

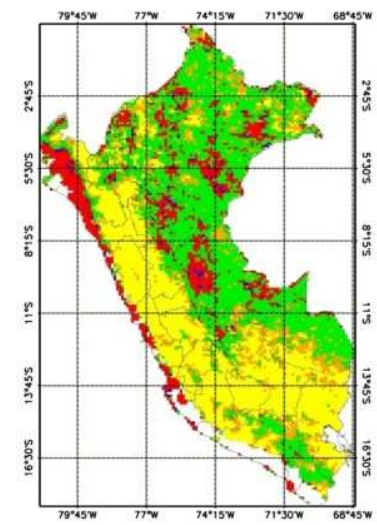

(c)

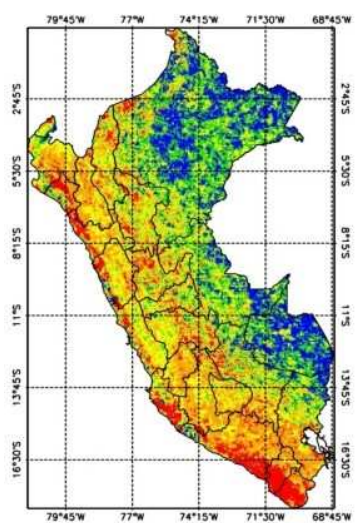

(e)

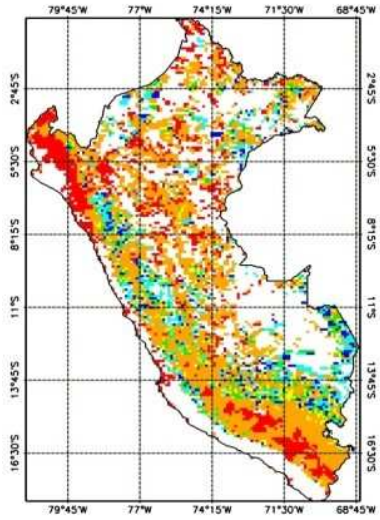

(b)

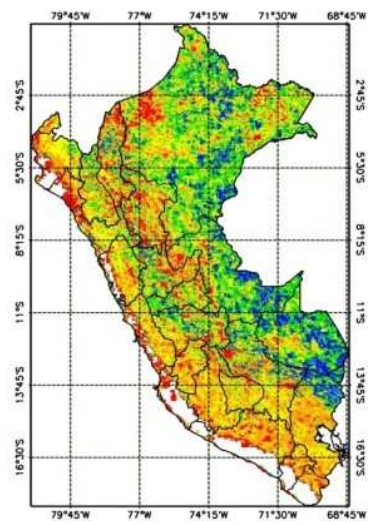

(d)

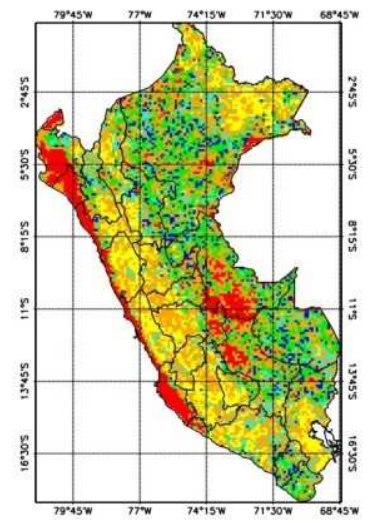

(f)

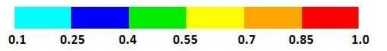

Figura 4: Se muestran el promedio mensual de los AOTs en la longitud de onda de $0.55 \mu \mathrm{m}$ para una resolución espacial de (a) 1 $\mathrm{km} \times 1 \mathrm{~km}$, (b) $10 \mathrm{~km} \times 10 \mathrm{~km}$ para el mes de abril del 2005, con datos de la NASA. El promedio anual de los AOTs en $0.55 \mu \mathrm{m}$ para una resolución espacial de (c) $1 \mathrm{~km} \times 1 \mathrm{~km}$, (d) $10 \mathrm{~km} \times 10 \mathrm{~km}$ para el año 2004 . El promedio anual de los AOTs en $0.55 \mu \mathrm{m}$ para el año 2005, con resolución espacial de (e) $1 \mathrm{~km} \times 1 \mathrm{~km}$ y (f) $10 \mathrm{~km} \times 10 \mathrm{~km}$. Los AOTs de resolución espacial de $1 \mathrm{~km} \times 1 \mathrm{~km}$ fueron obtenidos por SBDART [1], mientras que los de $10 \mathrm{~km} \times 10 \mathrm{~km}$ fueron proporcionados por la NASA, para todo el Perú. 
En la Figura 4 (a) y 4 (b) se visualiza el promedio mensual de las AOTs correspondiente al mes de abril del año 2005 estimado con una resolución espacial de $1 \mathrm{~km} \times 1 \mathrm{~km}$ y de $10 \mathrm{~km} \times 10 \mathrm{~km}$, respectivamente, con datos proporcionados por la NASA, para todo el Perú. En las Figuras 4(c) y $4(\mathrm{e})$ se visualiza el promedio anual de las AOTs SBDART para los años 2004 y 2005, respectivamente. Mientras que las Figuras 4(d) y 4(f) muestran el promedio anual de las

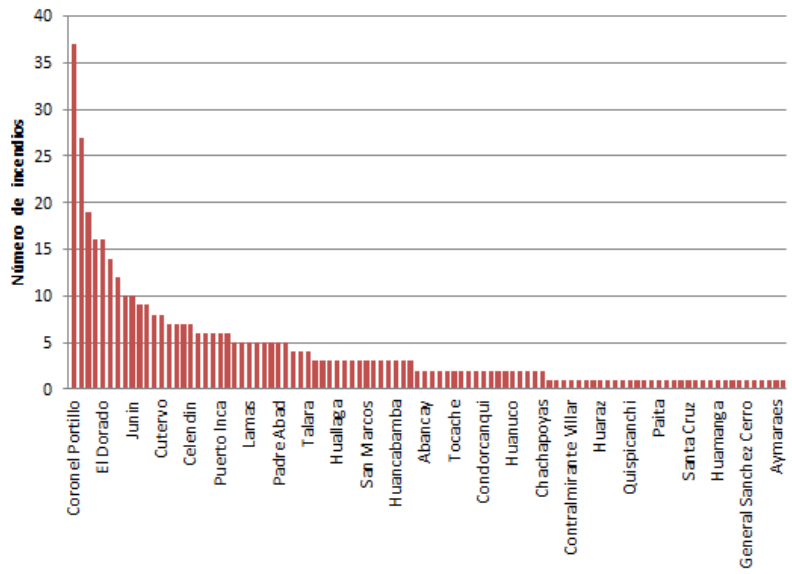

(a)

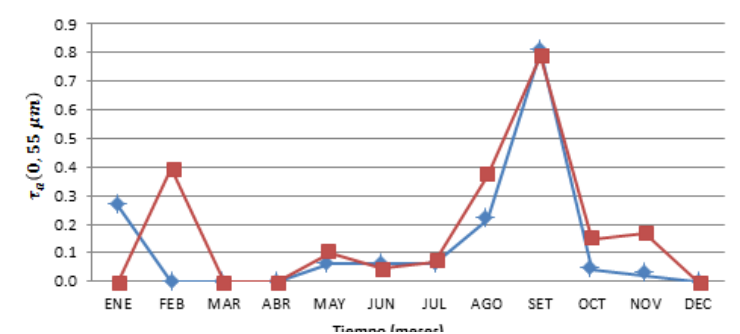

(c)

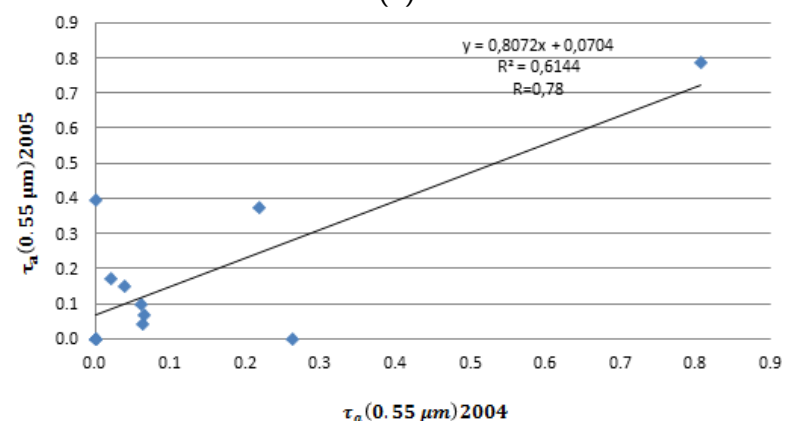

(e)
AOTs NASA para los años 2004 y 2005, respectivamente. El área se encuentra entre las latitudes $0^{\circ} 02^{\prime} 00^{\prime \prime}$ a $18^{\circ} 21^{\prime} 03^{\prime \prime}$ y las longitudes de $68^{\circ} 39^{\prime} 00^{\prime \prime}$ a $81^{\circ} 19^{\prime} 35^{\prime \prime}$. Las AOTs tienen valores en el rango de 0.1 a 1.0 , recordando que el AOT es adimensional.

En la Figura 5(a) y 5(b) se observa la cantidad de incendios forestales para las respectivas provincias del Perú durante los años 2004 y 2005, respectivamente.

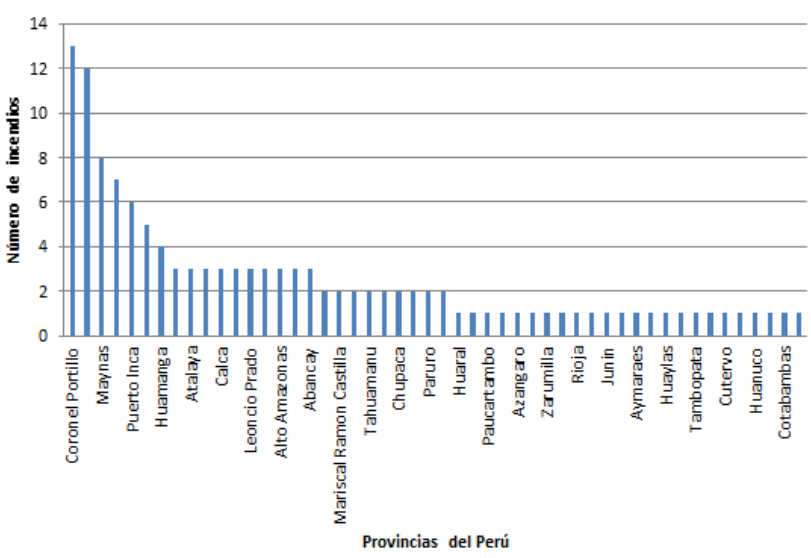

(b)

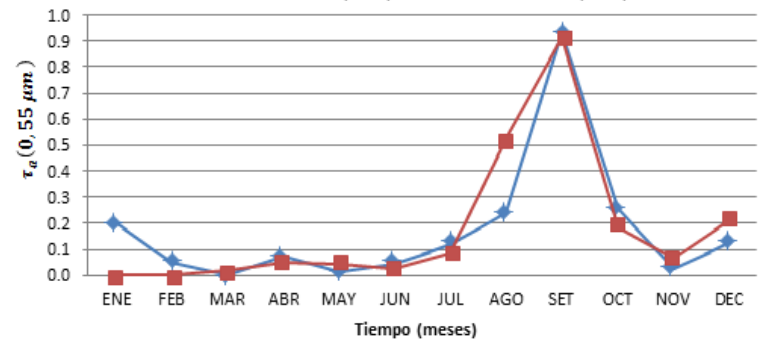

(d)

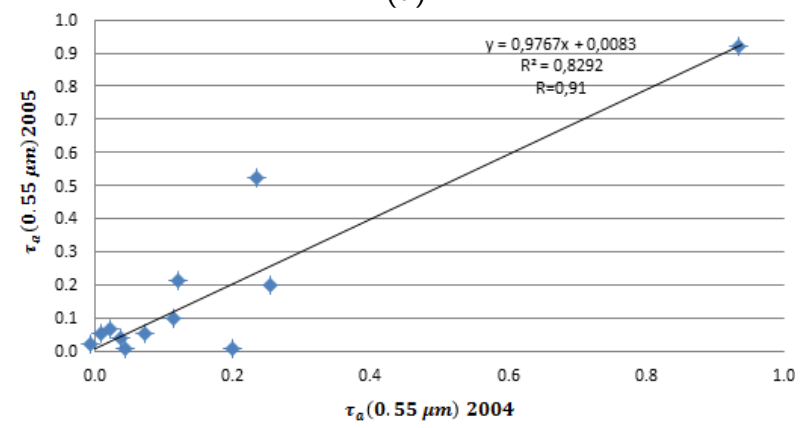

(f)

Figura 5: Incendios producidos en diferentes provincias del Perú para el año (a) 2004 y (b) 2005 [11]. Variación estacional de los AOTs SBDART correspondiente (c) Ucayali, y (d) Madre de Dios. Correlación del promedio mensual de los AOTs SBDART para los años 2004 y 2005, correspondientes a (e) Ucayali, y (f) Madre de Dios. 
En la Figura 5 (c) se muestra la distribución temporal de las AOTs SBDART correspondiente al departamento de Ucayali, ubicado en la coordenada central $10^{\circ} 24^{\prime} 22.00^{\prime \prime} \mathrm{S}$ y $72^{\circ} 35^{\prime} 40.26^{\prime \prime}$ O, para los años 2004 (azul) y 2005 (rojo), presentando valores máximos de 0.8 y 0.78 , para el mes de setiembre, respectivamente. En la Figura $5(d)$ se muestra la distribución temporal de los AOTs SBDART correspondiente al departamento de Madre de Dios, ubicado en la coordenada central $11^{\circ} 43^{\prime} 28.69^{\prime \prime} \mathrm{S}$ y $70^{\circ} 41^{\prime} 31.71^{\prime \prime} \mathrm{O}$, para los años 2004 (azul) y 2005 (rojo), presentando valores máximos de 0.9 y 0.92 pare el mes de setiembre, respecti- vamente. En la Figura 5(e), se muestra la dispersión de las AOTs SBDART para los años 2004 y 2005, correspondiente al departamento de Ucayali, ubicado en la coordenada central $10^{\circ} 24^{\prime} 22.00^{\prime \prime} \mathrm{S}$ y $72^{\circ} 35^{\prime} 40.26^{\prime \prime} \mathrm{O}$, presentando una correlación significativa $\approx 0.61$. Mientras que, en la Figura $5(\mathrm{f})$, se muestra la dispersión de los AOTs SBDART para los años 2004 y 2005, correspondiente al departamento de Madre de Dios, ubicado en la coordenada central $11^{\circ} 43^{\prime} 28.69^{\prime \prime} \mathrm{S}$ y $70^{\circ} 41^{\prime} 31.71^{\prime \prime} \mathrm{O}$, presentando una correlación significativa $\approx 0.83$.

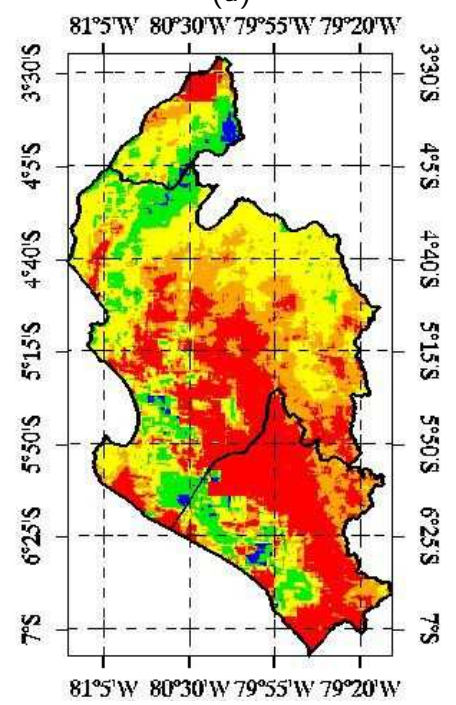

(c)

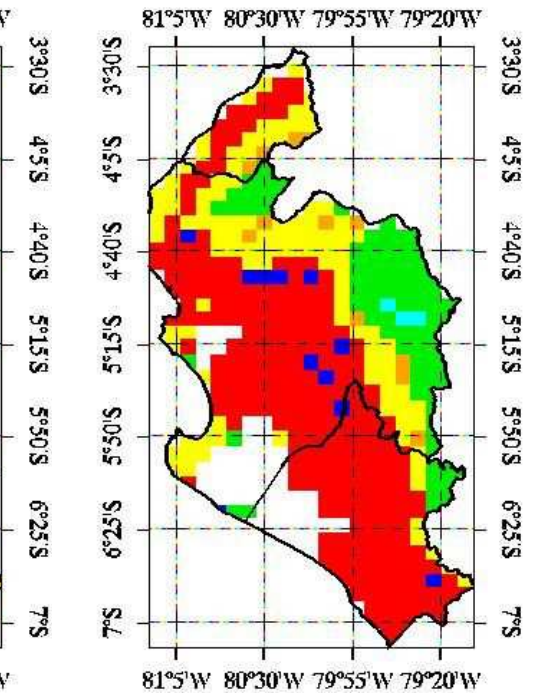

(b)

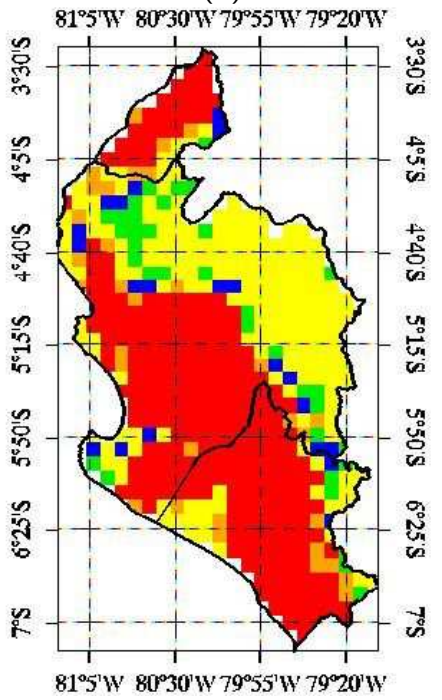

(d)

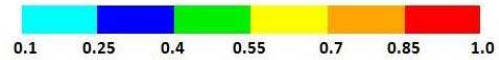

Figura 6: Las imágenes (a) y (c) muestran el promedio anual de los AOTs SBDART para la región norte del Perú, mientras que las imágenes (b) y (d) muestran el promedio anual de las AOTs NASA para los años 2004 y 2005. 


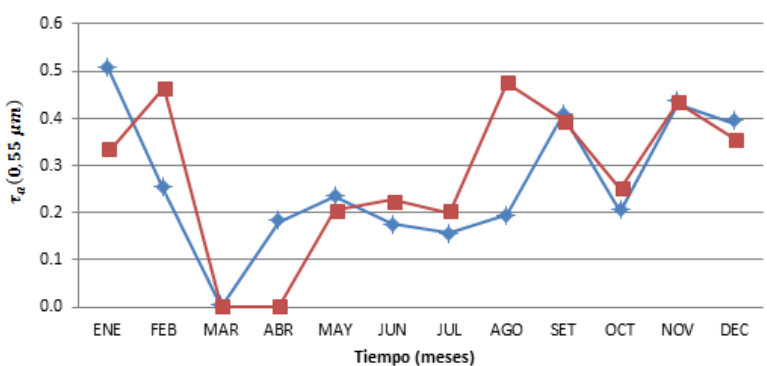

(a)

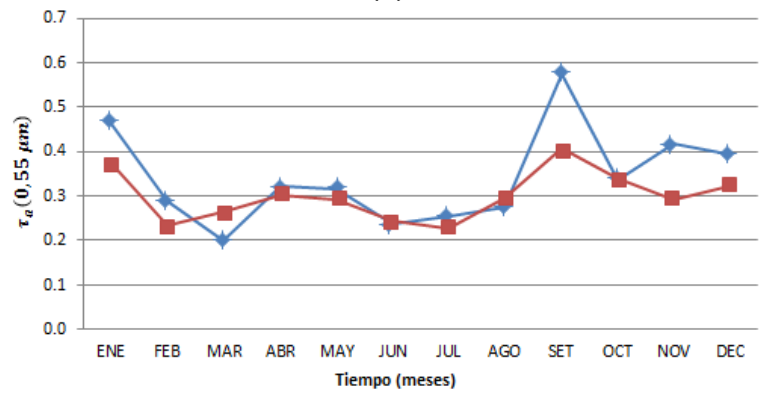

(c)

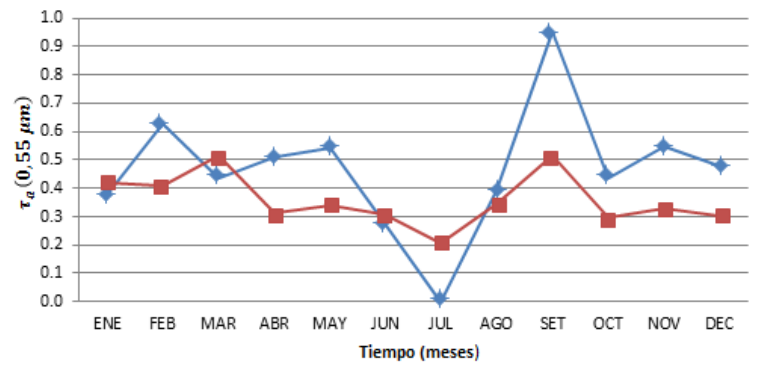

(e)

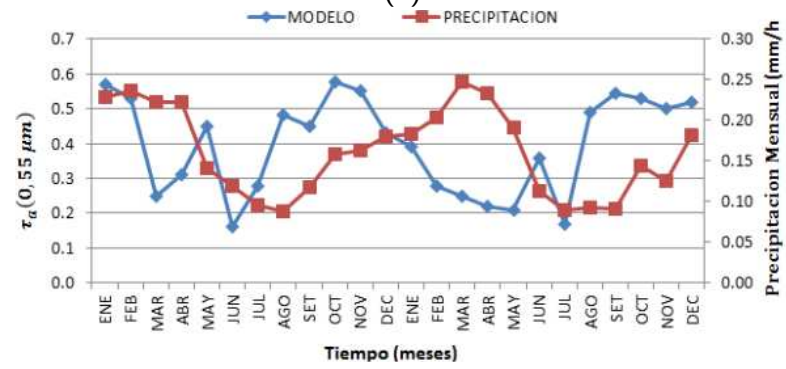

(g)

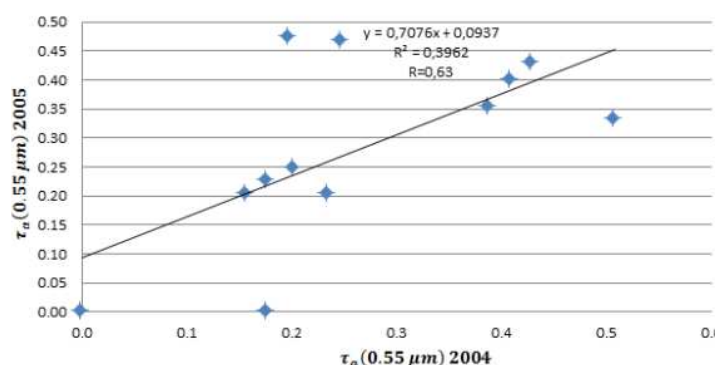

(b)

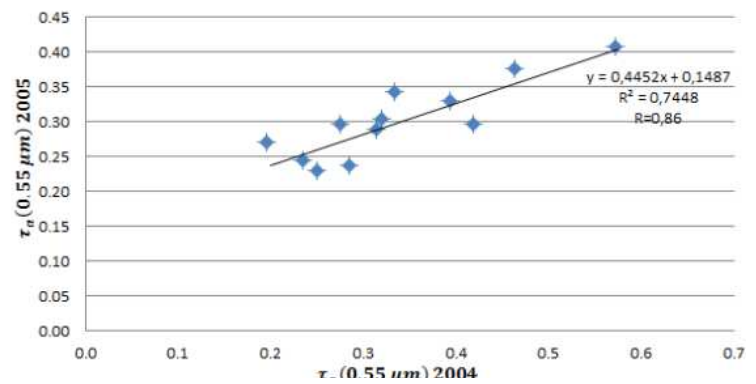

(d)

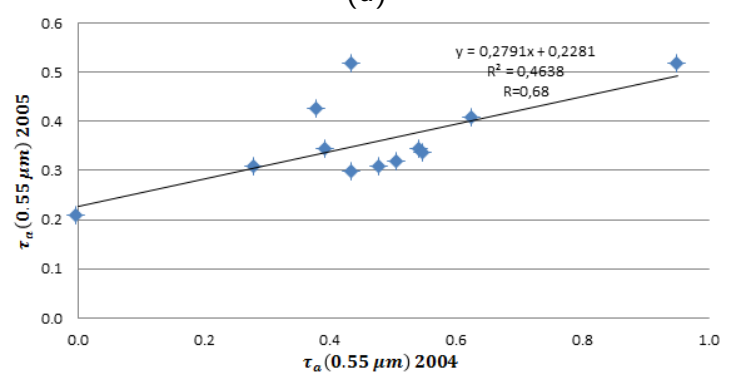

(f)

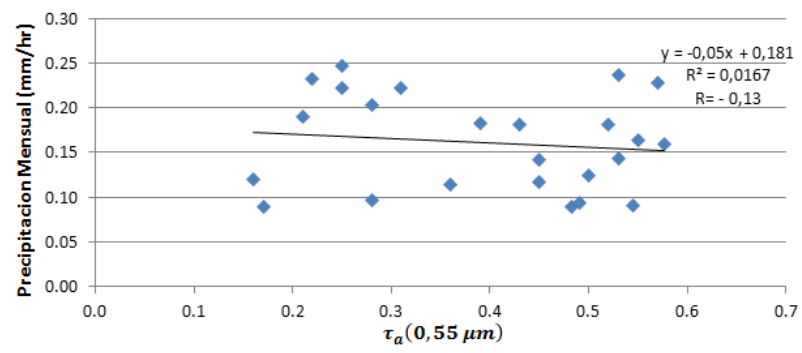

(h)

Figura 7: Se muestran la variación temporal de los AOTs SBDART correspondiente a (a) Tumbes, (c) Piura y (e) Lambayeque. La correlación de los promedios mensuales de los AOTs SBDART para los años 2004 y 2005 correspondiente a (b) Tumbes, (d) Piura y $(\mathrm{f})$ Lambayeque. En $(\mathrm{g})$ se muestra la variación estacional del AOTs SBDART correspondiente a la costa norte del Perú para los años 2004-2005, y la variación estacional de las precipitaciones durante el verano, noviembre-marzo, y en los meses invernales, abril-octubre; (h) muestra la correlación del promedio mensual del AOTs SBDART y las precipitaciones promedio mensual para los años 2004 y 2005.

La variabilidad espacial y temporal del AOTs SBDART que corresponden a la costa norte del Perú son mostradas a continuación. Los departamentos que sufren frecuente- mente los problemas de incendios forestales problema son Tumbes, Piura, Lambayeque en la costa norte, Cusco en la sierra su. En la última década San Martín, Ucayali y Madre 
de Dios en la región de la selva han mostrado un notable aumento de la superficie afectada por los incendios forestales [12]. En las Figuras 6(a) y 6(c), se muestran el promedio anual de las AOTs SBDART, mientras que en las Figuras 6(b) y 6(d) se muestran el promedio anual de los AOTs NASA para los años 2004 y 2005, respectivamente, para los departamentos de Tumbes, Piura, Lambayeque en la costa norte, ubicado en las coordenadas geográficas $3^{\circ} 24^{\prime}$ $\mathrm{S}-7^{\circ} 12^{\prime} \mathrm{S}$ y $81^{\circ} 19^{\prime} \mathrm{O}-79^{\circ} 16^{\prime} \mathrm{O}$.

En la Figura 7(a) se muestra la distribución temporal de las AOTs SBDART correspondiente al departamento de Tumbes, ubicado en la coordenada central $03^{\circ} 40^{\prime} 16^{\prime \prime} \mathrm{S}$ y $80^{\circ} 39^{\prime} 28^{\prime \prime}$ O para los años 2004 (azul) y 2005 (rojo), con valores en el rango de 0 a 0.5 y de 0 a 0.47 , respectivamente. En la Figura 7(c) se muestra la distribución temporal del AOT AOTs SBDART correspondiente al departamento de Piura, ubicado en la coordenada central $05^{\circ} 05^{\prime} 15^{\prime \prime} \mathrm{S}$ y $81^{\circ} 06^{\prime} 49^{\prime \prime} \mathrm{O}$ para los años 2004 (azul) y 2005 (rojo), con valores en el rango de 0.2 a 0.57 y de 0.22 a 0.40 , respectivamente. En la Figura $7(\mathrm{e})$ se muestra la distribución temporal del AOTs SBDART correspondiente al departamento de Lambayeque, ubicado en la coordenada central $06^{\circ} 01^{\prime} 42^{\prime \prime} \mathrm{S}$ y $80^{\circ} 0^{\prime} 58^{\prime \prime} \mathrm{O}$ para los años 2004 (azul) y 2005 (rojo), con valores en el rango de 0 a 0.95 y de 0.21 a 0.51 , respectivamente.

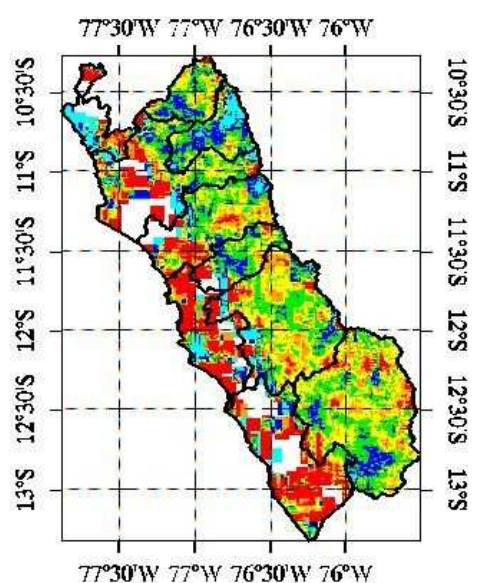

(a)

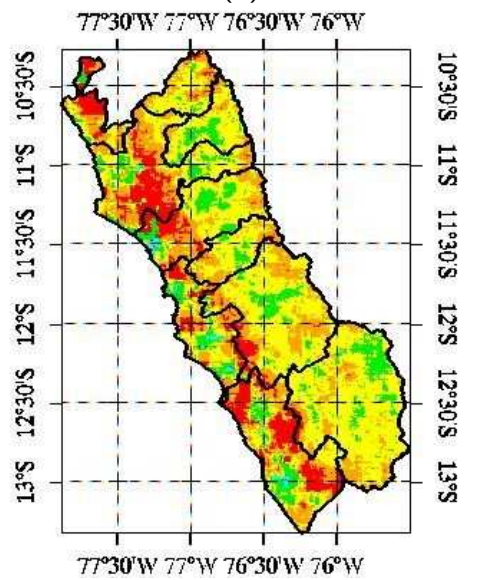

(c)

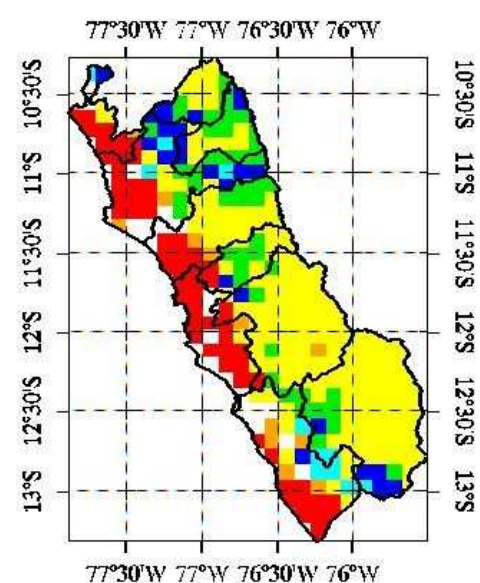

(b)

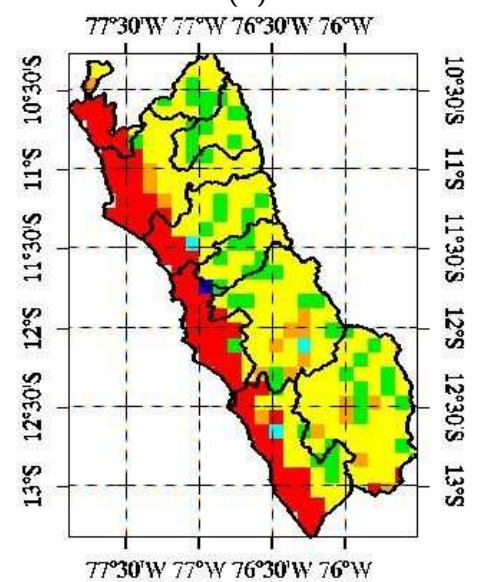

(d)

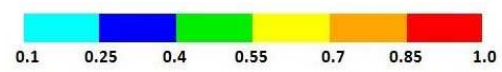

Figura 8: Las imágenes (a) y (c) muestran el promedio anual de los AOTs SBDART para el Departamento de Lima, mientras que las imágenes (b) y (d) muestran los AOTs NASA para los años 2004 y 2005, respectivamente. 


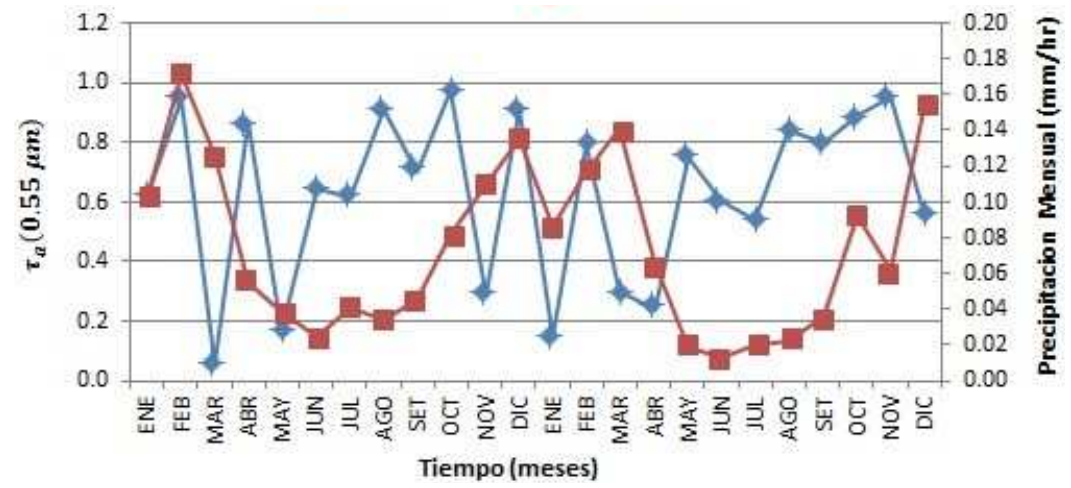

(a)

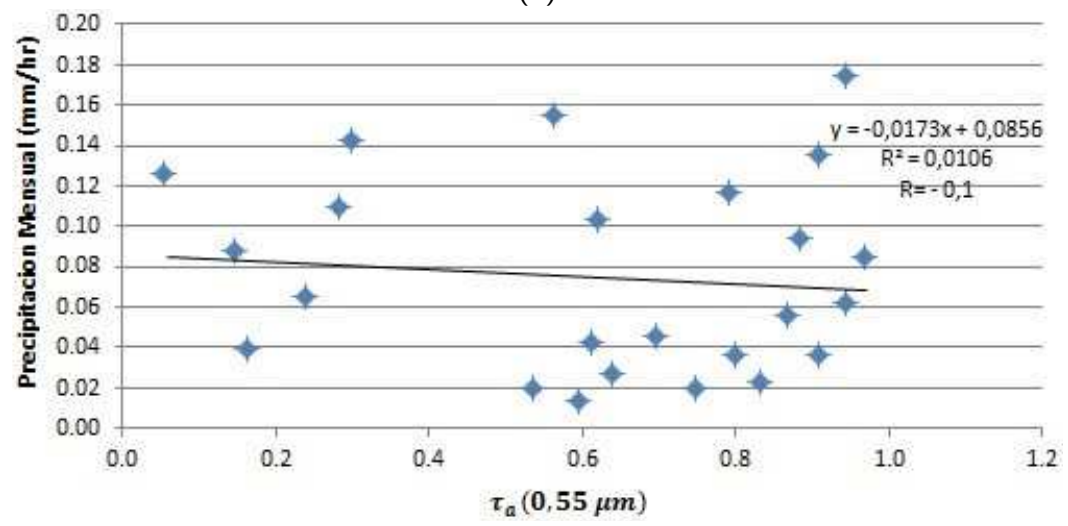

(b)

Figura 9: (a) Distribución temporal de los AOTs SBDART correspondiente al departamento de Lima para los años 2004 y 2005 , en color azul, así como la distribución temporal de las precipitaciones durante el verano ocurrida entre noviembre y marzo y en los meses invernales de abril a octubre, en color rojo. (b) Correlación del promedio mensual de los AOTs SBDART y del promedio mensual de las precipitaciones, para los años 2004 y 2005.

En la Figura 7(b) se muestra la gráfica de dispersión de los AOTs SBDART para los años 2004 y 2005, correspondiente al departamento de Tumbes presentando una correlación significativa $\approx 0.39$. Mientras que en la Figura $7(\mathrm{~d})$ se muestra la gráfica de dispersión de los AOTs SBDART para los años 2004 y 2005, correspondientes al departamento de Piura, presentando una correlación significativa $\approx 0.74$. Y finalmente en la Figura $7(f)$ se muestra la gráfica de dispersión de las AOTs SBDART para los años 2004 y 2005, correspondiente al departamento de Lambayeque presentando una correlación significativa $\approx 0.46$.

En la Figura $7(\mathrm{~g})$, se muestra la distribución temporal de los AOTs SBDART en el rango de 0.16 a 0.58 y la distribución temporal de las precipitaciones medidos en $\mathrm{mm} / \mathrm{hr}$, durante el verano, noviembre-marzo y en los meses invernales, abril-octubre, correspondiente a la costa norte del Perú para los años 2004 (azul) y 2005 (rojo), en la cual se observa que los valores altos de precipitación ocurrieron cuando los valores de los AOTs fueron los más bajos, esto es debido a que durante el verano, noviembre-marzo, es la estación en donde se presenta la mayor actividad convectiva, la Zona de Convergencia Intertropical, ZCIT, en la que el Pacífico ecuatorial oriental se desplaza hacia una posición más austral, $1^{\circ} \mathrm{N}$, y en los meses invernales, abril-octubre, se aleja de la línea ecuatorial hasta alcanzar su posición más norte, $10^{\circ} \mathrm{N}$. Entre diciembre y marzo, la ZCIT migra hacia el sur ocasionando precipitaciones sobre Ecuador y alcanzando la costa norte del Perú [13]. En las Figura 7(h) se muestra la gráfica de la dispersión del promedio mensual de las AOTs CBDART para los años 2004 y 2005, y el promedio mensual de las precipitaciones, en $\mathrm{mm} / \mathrm{hr}$, mostrando una baja correlación, entre ellas.

También hemos estudiado la variabilidad espacial y temporal de los AOTs SBDART correspondientes al departamento de Lima. En las Figuras 8(a) y 8(c) se muestran el promedio anual de los AOTs SBDART, mientras que en las Figuras $8($ b) y $8(d)$ se muestran el promedio anual de los AOTs NASA para los años 2004 y 2005, respectivamente, considerando que el departamento de Lima está ubicado en la coordenada central $12^{\circ} 03^{\prime} 05^{\prime \prime} \mathrm{S}$ y $77^{\circ} 01^{\prime} 54^{\prime \prime} \mathrm{O}$. 


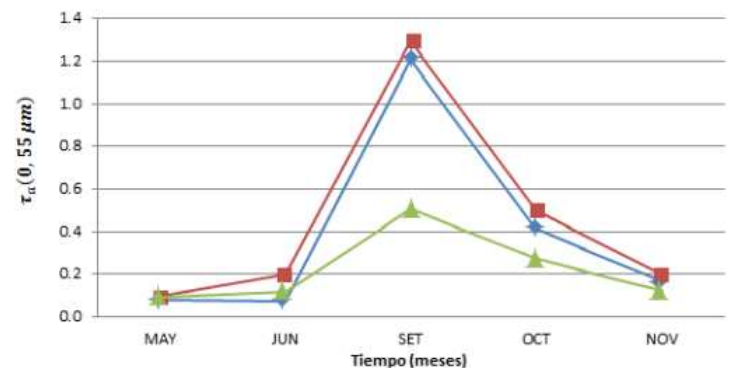

(a)

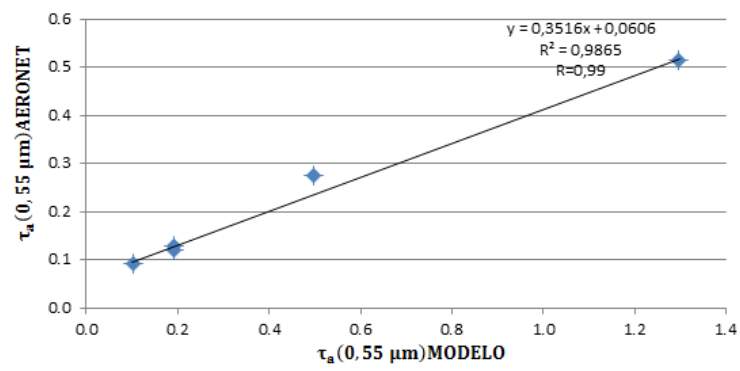

(c)

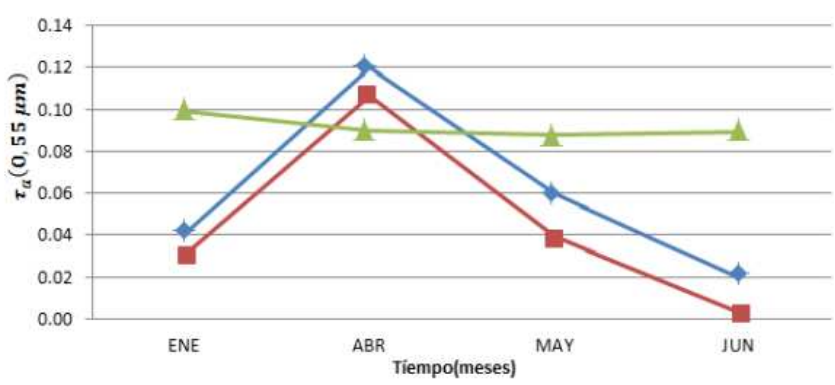

(e)

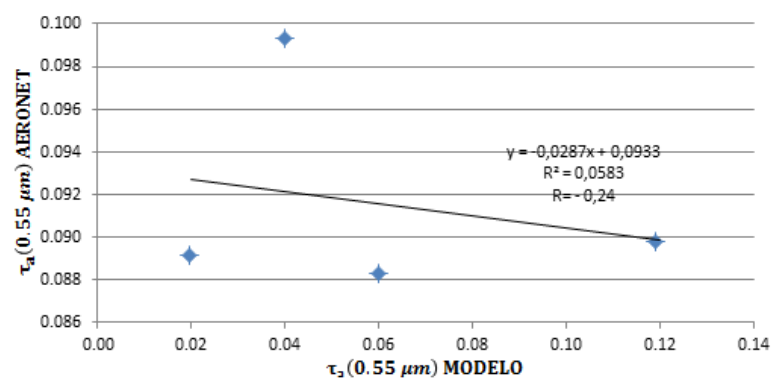

(g)

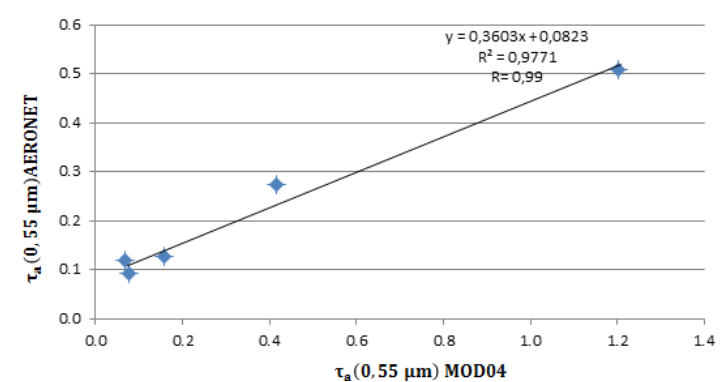

(b)

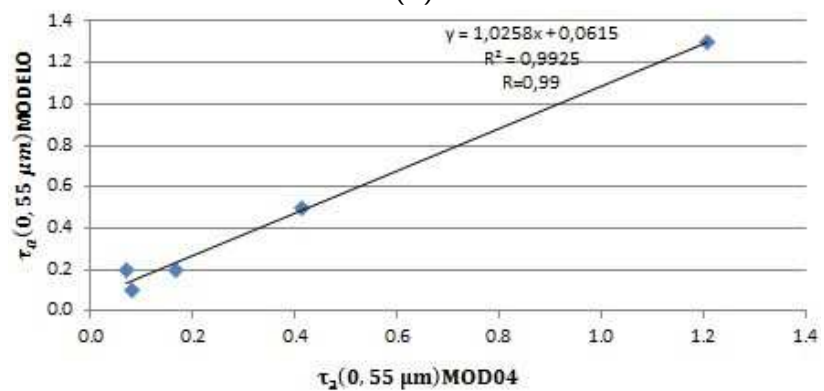

(d)

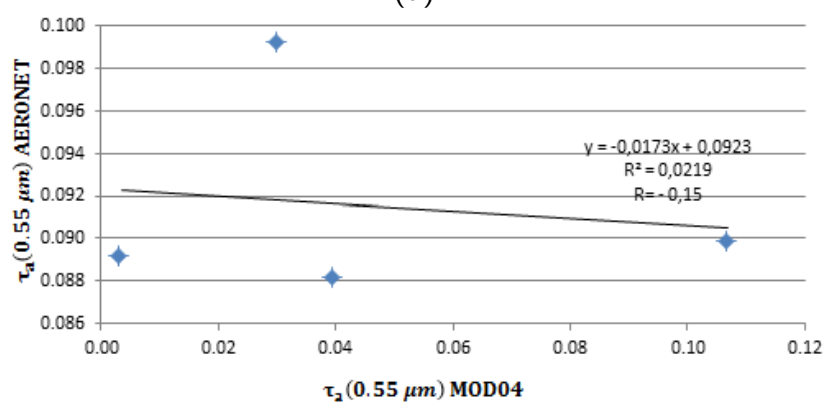

(f)

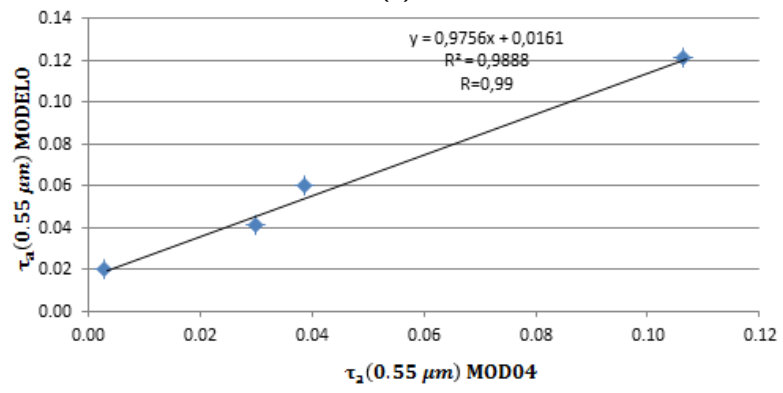

(h)

Figura 10: Se muestran (a) los promedios mensuales de los AOTs AERONET con resolución espacial de $1 \mathrm{~km} \times 1 \mathrm{~km}$ (color rojo) y de $10 \mathrm{~km} \times 10 \mathrm{~km}$ (color azul); (b) la correlación de los promedios mensuales de los AOTs AERONET con resolución de $10 \mathrm{~km} \times 10$ km; (c) la correlación de los promedios mensuales de los AOTs AERONET para $1 \mathrm{~km} \times 1 \mathrm{~km}$ de resolución espacial; (d) la correlación de los promedios mensuales de los AOTs ERONET de $1 \mathrm{~km} \times 1 \mathrm{~km}$ y de $10 \mathrm{~km} \times 10 \mathrm{~km}$; (e) los promedios mensuales de los AOTs AERONET con $1 \mathrm{~km} \times 1 \mathrm{~km}$ (color azul) y con $10 \mathrm{~km} \times 10 \mathrm{~km}$ (color rojo); (f) la correlación del promedio mensual de los AOTs AERONET para $10 \mathrm{~km} \times 10 \mathrm{~km}$ de resolución espacial; (g) la correlación del promedio mensual de los AOTs de $1 \mathrm{~km} \times 1 \mathrm{~km}$ y (h) la correlación del promedio mensual de los AOTs de $1 \mathrm{~km} \times 1 \mathrm{~km}$ y $10 \mathrm{~km} \times 10 \mathrm{~km}$ de resolución espacial procedente del sensor MODIS. 
En las dos últimas figuras mencionadas se visualiza las altas concentraciones de los AOTs cerca del litoral peruano, debido a las partículas de sal marina generadas en el mar por la acción mecánica del viento o la lluvia sobre su superficie, desprendiendo burbujas, que al explotar inyectan pequeñas gotas de agua salada en el aire. Estas gotas de agua, bajo determinadas condiciones de humedad, pueden quedar en suspensión en la atmósfera, y cuando la humedad disminuye produce una evaporación de estas pequeñas gotas obteniéndose partículas salinas sólidas de $\mathrm{NaCl}$ [2]

En la Figura 9(a) se muestra la distribución temporal del espesor de los AOTs SBDART correspondiente al departamento de Lima para los años 2004 y 2005 con valores en el rango de 0.06 a 0.97 , así como la distribución temporal de las precipitaciones durante el verano, noviembre-marzo, y en los meses invernales, abril-octubre, los valores altos de los AOTs SBDART ocurrieron cuando los valores precipitación fueron los más bajos, los valores altos de los AOTs SBDART en la zona Metropolitana de Lima y Callao se debe al sector transporte, tal como se estimó en el año 2001, por el entonces Consejo Nacional del Ambiente, hoy el actual Ministerio del Ambiente, MINAM, al realizar el inventario de las emisiones atmosféricas totales, donde se identificó que los aportes del sector transporte e industria significaron el $86 \%$ y $14 \%$ del inventario, respectivamente [14]. En las Figura 9(b) se muestra la gráfica de la dispersión entre el promedio mensual de las AOTs SBDART los años 2004 y 2005, y el promedio mensual de las precipitaciones en $\mathrm{mm} / \mathrm{hr}$, mostrando una baja correlación.

Finalmente, en la Figura 10(a), se muestran las series de tiempo para analizar la variabilidad temporal de las AOTs para el año 2004, para la estación de la red AERONET de Rio Branco, Brasil. Se identificaron los meses de setiembre y octubre como los meses que presentan mayor valor del AOT AERONET (color verde) de 0.51 y 0.27 , respectivamente. Esto es debido a la quema de biomasa que hay en el Brasil. Para los meses de enero, febrero, marzo, abril, julio, agosto y diciembre, no se contó con registros por parte de esa estación. Para los meses de setiembre y octubre se registraron valores medios mensuales del AOT NASA (color azul) de 1.2 y 0.4 , respectivamente, procedentes del producto MOD04_L2 de aerosoles medidos en $10 \mathrm{~km} \times 10$ $\mathrm{km}$ de resolución espacial; de igual manera, para los meses de setiembre y octubre se determinaron los valores medios mensuales del AOT SBDART (color rojo) de 1.3 y 0.5 , respectivamente.

La Figura 10(b) se muestra la gráfica de la dispersión del AOT NASA respecto al AOT AERONET presentando una correlación significativa de $\approx 0.98$, esto para el año 2004. Mientras que la Figura 10 (c), muestra la gráfica de la dispersión entre el AOT SBDART respecto al AOT AERONET presentando una correlación significativa de approx 0.99. Y finalmente, la Figura 10 (d) muestra la gráfica de la dispersión entre el AOT NASA y el AOT SBDART presentando una correlación significativa de approx 0.99, esto para el año 2004.

En la Figura $10(\mathrm{e})$, se muestran las series de tiempo para analizar la variabilidad temporal del AOT para el año 2005, dentro de la región de estudio. Se identificaron los meses de enero y abril como los meses con máximos valores de las medias mensuales del AOT AERONET de 0.099 y 0.090 , respectivamente. Para los meses de febrero, marzo, julio, agosto, setiembre, octubre, noviembre, diciembre, no se contó con registros por parte de la estación de AERONET. Se registró para el mes de abril valores medios mensuales del AOT NASA y por ende para el AOT SBDART.

Para el año 2005, la Figura 10(f) muestra la gráfica de la dispersión del AOT NASA respecto al AOT AERONET presentando una correlación no significativa de approx 0.02. Mientras que, la Figura $10(\mathrm{~g})$ muestra la gráfica de la dispersión entre los AOT SBDART y los AOTs AERONET, presentando una correlación no significativa de approx 0.06 . Finalmente la Figura $10(\mathrm{~h})$ muestra la gráfica de la dispersión entre los AOTs NASA y los AOTs SBDART, presentando una correlación significativa de $\approx 0.99$.

\section{Conclusiones}

Hemos estimado el espesor óptico de los aerosoles en la longitud de onda de $0.55 \mu \mathrm{m}$ con una resolución espacial de $1 \mathrm{~km} \times 1 \mathrm{~km}$, mediante el SBDART [1, a partir de las ecuaciones inversas generadas mediante una regresión polinomial de grado 3, la cual corresponde a la menor reflectancia de la superficie terrestre en la longitud de onda de $0.66 \mu \mathrm{m}$ correspondiente a la banda 1 de las imágenes Level $1 \mathrm{~B}(\bmod 021 \mathrm{~km})$ del sensor MODIS), validados con el producto MODIS (mod04 L2) de aerosoles y con los datos medidos in situ provenientes de la estación de la red AERONET ubicada en Rio Branco, Brasil.

Los valores del espesor óptico de los aerosoles a 0.55 $\mu \mathrm{m}$ para los meses de agosto y septiembre son altos cerca de la región amazónica tanto para el año 2004 y 2005. Esto coincide con los tiempos del inicio de los incendios forestales en Brasil.

En la costa norte del Perú, los valores altos de la precipitación ocurrieron cuando los valores del espesor óptico de los aerosoles a $0.55 \mu \mathrm{m}$ fueron los más bajos.

El modelo SBDART utilizado para la estimación del espesor óptico de los aerosoles a $0.55 \mu \mathrm{m}$ en algunas regiones del Perú, se debe a la existencia de datos sobre su ubicación geográfica, las condiciones meteorológicas, la circulación atmosférica y a las propiedades ópticas de los aerosoles, puesto que en el Perú no tenemos un fotómetro CIMEL perteneciente a la red AERONET, por lo que es necesario implementar tres, por los menos, para obtener datos in situ para la costa, sierra y selva. 


\section{Agradecimientos}

E. Ch. agradece al Programa Cátedra CONCYTEC por el soporte financiero de la beca de Maestría en Física.

\section{Referencias}

[1] P. Ricchiazzi y S. Yang; Bull. Am. Meteorol. Soc 79, 2101 (1998)

[2] E. R. Martínez. Estudio de los aerosoles atmosféricos en Valencia mediante medidas del Nefelómetro de integración; pp. 7-8; Tesis para optar el grado de Doctor en Ciencias Físicas, Universidad de Valencia, Valencia (2011).

[3] Consejo Interamericano para el Desarrollo Integral, ClDI. Evaluación de los niveles de contaminación atmosférica en las zonas urbanas del valle de Aburra, Universidad Pontificia Bolivariana, Medellín, Colombia (2010).

[4] Intergovernmental Panel on Climate Change, IPCC. Climate Change 2007: The Physical Science Basis. Contribution of Working Group I to the Fourth Assessment Report of the Intergovernmental Panel on Climate Change, Cambridge University Press, Cambridge, New York, UK and USA (2007).

[5] World Health Organization, WHO. (2006). Air quality guidelines for particulate matter, ozone, nitrogen dioxide and sulfur dioxide. Global update 2005. Summary of risk assessment. WHO Press, Ginebra, Suiza (2006).
[6] L. Suárez, L. Castillo, M. Marín, G. Carrillo, L. Rimac, J. Pomalaya y R. Menacho; Mosaico Científico 3(3), 36 (2006).

[7] K. N. Liou; An Introduction to Atmospheric Radiation, Second Edition, pp. 206-301, Academic Press, New York, USA (2002).

[8] W. E. Meador y W. R. Weaver; J. Atmos. Science. 37, 630, (1990).

[9] http://ladsweb.nascom.nasa.gov/data/search.html

[10] A. D. A. Castanho; Propiedades ópticas das partículas de aerosol e uma nova metodologia para a obtenção da espesssura óptica via saélite sobre São Paulo, Tese de doutorado, Instituto de Física, Universidade de São Paulo, São Paulo, Brasil (2005).

[11] http://www.dpi.inpe.br/proarco/bdqueimadas/.

[12] M. I. Manta y H. León; Floresta 34(2), 179 (2004).

[13] N. Quispe, F. H. Cubas y G. J. Avalos; Memoria Técnica, Componente Meteorológica-SENHAMIPerú, pp.2-3 (2005).

[14] Comisión Nacional del Ambiente, CONAM; Inventario de Emisiones Totales, Lima, Perú (2001). 